\title{
Assessment of Stacking Faults in Silicon Carbide Crystals
}

\author{
Hidekazu Yamamoto* \\ Faculty of Engineering, Chiba Institute of Technology, \\ 2-17-1 Tsudanuma, Narashino, Chiba 275-0016, Japan \\ (Received September 24, 2012; accepted November 5, 2012)
}

Key words: silicon carbide ( $\mathrm{SiC})$, staking fault, X-ray topography, photoluminescence (PL), mirror electron microscopy (MEM), atomic force microscopy (AFM)

X-ray topography, photoluminescence (PL) spectroscopy, mirror electron microscopy (MEM), and atomic force microscopy (AFM) were employed to evaluate stacking faults in silicon carbide ( $\mathrm{SiC}$ ) crystals. The results reveal that transmission X-ray topography can be used to assess internal stacking faults in crystals, while PL spectroscopy and MEM can be used to assess stacking faults near the surface. The stacking faults assessed by these different methods were found to be the same. AFM revealed that sites where stacking faults were exposed on the surface had microscopic level differences of about 0.1 $\mathrm{nm}$, which are thought to be generated by different etch rates during mirror polishing.

\section{Introduction}

Silicon carbide ( $\mathrm{SiC})$, which represents a class of wide-bandgap semiconductors, is a highly promising crystalline material for power devices ${ }^{(1)}$ and it is expected to replace single-crystal $\mathrm{Si}$ in next-generation power devices. For other use, $\mathrm{SiC}$ is expected as an optoelectronic material in ultraviolet (UV) or X-ray detector. ${ }^{(2)}$ However, it is difficult to grow single-crystal $\mathrm{SiC}$ from the liquid phase. Consequently, the mass production of $\mathrm{SiC}$ is based on sublimation. However, sublimation employs wafer-shaped seed crystals, which makes it difficult to produce large wafers and to reduce the number of crystal defects. Some crystal defects are fatal to devices, whereas others have no adverse effects. ${ }^{(3)}$ In the development of $\mathrm{SiC}$ crystal manufacturing technologies, it is important to develop methods of detecting and reducing defects that are detrimental to devices. Stacking faults in $\mathrm{SiC}$ have been reported to degrade the characteristics of bipolar devices. ${ }^{(4)}$ It is thus critical to develop methods of assessing stacking faults for practical applications of $\mathrm{SiC}$ power devices.

${ }^{*}$ Corresponding author: e-mail: yamamoto.hidekazu@it-chiba.ac.jp 
This study uses X-ray topography, photoluminescence (PL) spectroscopy, mirror electron microscopy (MEM), and atomic force microscopy (AFM) to assess stacking faults in $\mathrm{SiC}$ crystals. The results reveal that transmission-mode X-ray topography can be used to assess internal stacking faults in crystals. PL spectroscopy and MEM can be used to assess stacking faults at crystal surfaces. As a result, these methods were used to assess the same stacking faults. Assessment by AFM revealed the presence of microscopic level differences of about $0.1 \mathrm{~nm}$ at sites where stacking faults were exposed on the surface.

\section{Materials and Methods}

$4^{\circ}$-offset $4 \mathrm{H}-\mathrm{SiC}(0001)$ surface n-type 3 -inch wafers were used to assess crystal defects. These wafers were approximately $360 \mu \mathrm{m}$ thick. Sublimation was used to grow crystals with a resistivity in the range of $0.015-0.025 \Omega \cdot \mathrm{cm}$.

BedeScan (Bede Scientific Instruments) with a Mo K-alpha X-ray source was employed for X-ray topography measurements. Transmission mode was used for the diffraction plane (1-100). The transmission mode enabled the defect distribution to be analyzed throughout the interior of the wafer.

A PL imaging analyzer (PLI-100, Photon Design Corp.) was employed for PL spectroscopy assessment. The excitation light was generated by a $313 \mathrm{~nm}$ laser. Luminescence at wavelengths of 420 and $750 \mathrm{~nm}$ or above was analyzed.

For MEM assessment, a microscope fabricated by Hitachi Ltd., Central Research Laboratory was employed. Like scanning electron microscopy (SEM) and transmission electron microscopy (TEM), MEM employs an electron beam as the probe, but unlike SEM and TEM, MEM applies a negative bias voltage to cause the electron beam to reflect and to form an image by imaging the reflected electron beam. ${ }^{(5)}$ The reflected direction of the electron beam varies according to the electric potential or surface roughness of the sample. Thus, the electric potential distribution and/or microscopic level differences on sample surfaces can be analyzed. In addition, UV irradiation can generate carriers in the sample and the change in the potential due to defects trapping the carriers can be analyzed. This system can be used to analyze wafer samples and defects detected by other assessment methods with its coordinate identification function.

Level difference analysis was performed by AFM (XE-HDM, Park Systems Corp.). This microscope can be used to analyze wafer samples in the same manner as the MEM system and it can also be used to analyze defects detected by other assessment methods with its coordinate identification function.

\section{Results}

\subsection{Assessment by X-ray topography}

Figure 1 shows the assessment results for $\mathrm{SiC}$ wafers obtained by transmission-mode $\mathrm{X}$-ray topography. The overall crystallinity is good, but defects are visible in the lower left of the image as lines in the $\langle 1-100\rangle$ direction. Figure 2 shows a state of stacking fault running from the rear surface to the front surface. By assuming that the analyzed 


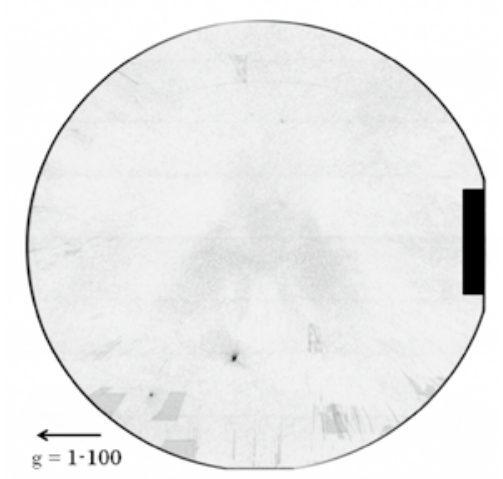

Fig. 1. Assessment result by transmission X-ray topography.

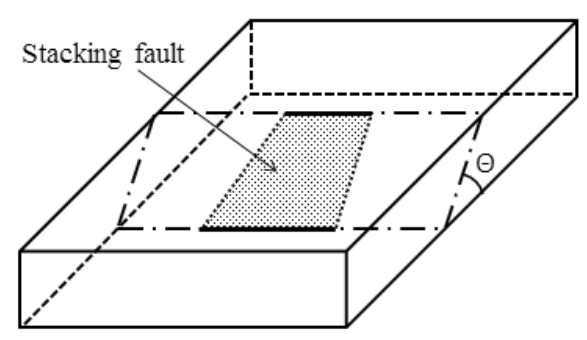

(a)



(b)

Fig. 2. State of stacking fault running from rear surface to front surface: (a) bird's-eye view and (b) cross section.

defects are stacking faults, the following formula can be used to calculate the length $d$ of the projected defect under the assumption that the stacking fault extends from the rear surface to the front surface of the wafer.

$$
d=t / \tan \theta
$$

Here, $t$ is the wafer thickness and $\theta$ is the offset angle. The wafers used in the analysis are approximately $360 \mu \mathrm{m}$ thick and have a crystal offset angle of $4^{\circ}$. Calculating $d$ using eq. (1) gives $5.15 \mathrm{~mm}$. The actual length of defect $\mathrm{A}\left(d_{\mathrm{A}}\right)$ in Fig. 3 is approximately $5 \mathrm{~mm}$. This result is reasonable if $\operatorname{defect} \mathrm{A}$ is a stacking fault. 


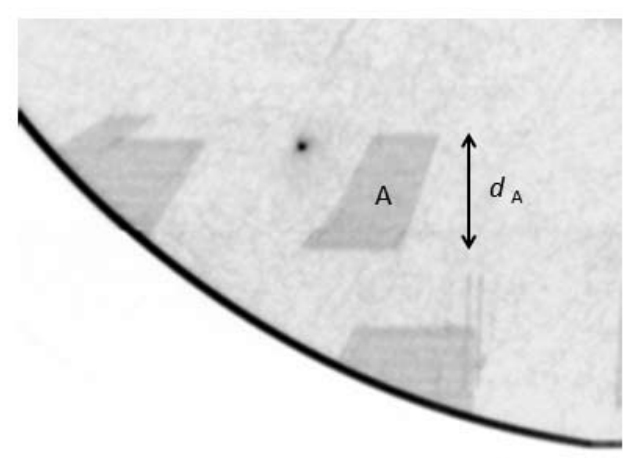

Fig. 3. Length of defect.

\subsection{Assessment by PL spectroscopy}

According to previous studies, PL spectroscopy can be used to detect stacking faults in $\mathrm{SiC}$ crystals; single and multilayer stacking faults are detected by luminescence at approximately 420 and $510 \mathrm{~nm}$, respectively. ${ }^{(6)}$

Figure 4 shows PL spectroscopy assessment for luminescence at a wavelength of $750 \mathrm{~nm}$ or above. No luminescence originating from defects is observed in the section detected by X-ray topography. However, there is a circular portion slightly above the center of the wafer that exhibits weak luminescence; it is thought to be caused by a difference in dopant concentration. The measurement results also exhibit a pattern that is apparently caused by variable CCD sensitivity over the analyzed area.

Figure 5 shows luminescence at $420 \mathrm{~nm}$. Linear emissions were observed at the site where defects were detected by X-ray topography (indicated by white arrows in the figure). The emissions are thought to be caused by single-layer stacking faults. PL spectroscopy detects the linear emissions due to emissions solely from the wafer surface.

The assessment results obtained by X-ray topography and $420 \mathrm{~nm}$ PL spectroscopy are shown in Fig. 6. The alignment is quite satisfactory, with PL spectroscopic emissions observed along the lines in one direction for the defects detected by X-ray topography. These results can be explained as stacking faults detected by X-ray topography, which extend from the rear surface through the front surface, where PL spectroscopic emissions are generated by defects on the front surface. Thus, X-ray topography is considered to have detected single-layer stacking faults. However, the PL spectroscopic emission along the lines $\mathrm{f}-\mathrm{g}$ in Fig. 6 was not detected by X-ray topography. Although this emission is also considered to originate from a single-layer stacking fault, it may not extend from the rear surface to the front surface. Further detailed evaluation is necessary.

\subsection{MEM assessment}

MEM was employed to assess the sites with luminescence caused by single-layer stacking faults as detected by PL spectroscopy. Figure 7 shows the analysis results for UV irradiation at points a-e, h, and i in Fig. 6. Linear defects were detected at all 



Fig. 4 (left). PL assessment result (> $750 \mathrm{~nm})$.

Fig. 5 (right). PL assessment result $(420 \mathrm{~nm})$.

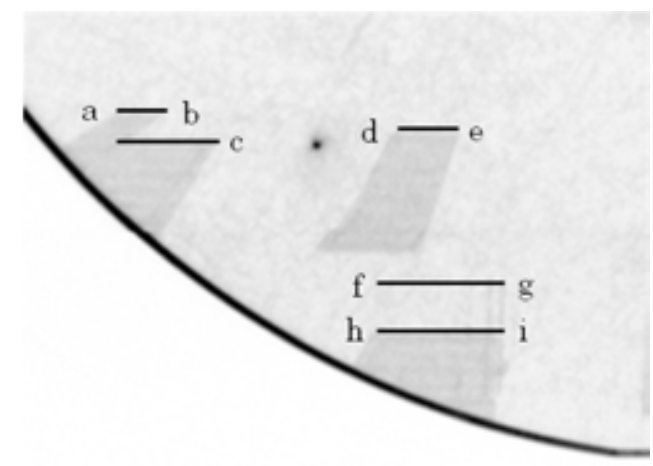

Fig. 6. Comparison of X-ray topography and PL assessment results (420 nm).

measurement points. Thus, MEM is also able to detect stacking faults on wafer surfaces and can assess the incursion of stacking faults into the wafer's interior.

Figure 8 compares MEM assessment for the incursion of stacking faults from the top surface to the interior of the wafer with X-ray topography assessment. The two measured values at point $\mathrm{h}$ are in the range of $116-117^{\circ}$, which agree well. Table 1 lists the measured values for the angle of incursion into the wafer interior for stacking faults according to MEM and X-ray topography measurements for points a-e, h, and i. These values agree well, suggesting that both methods assessed the same stacking faults. 


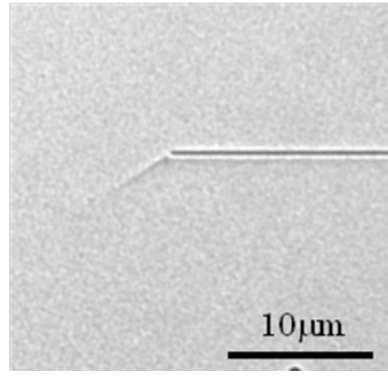

(a)



(c)

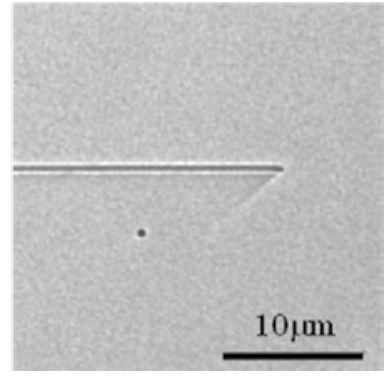

(b)

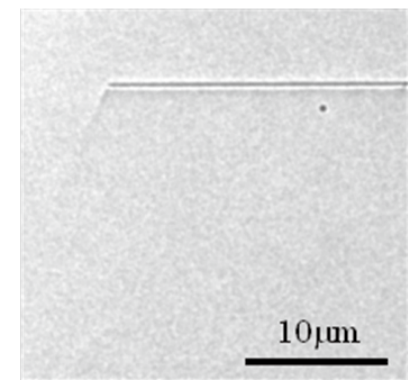

(d)

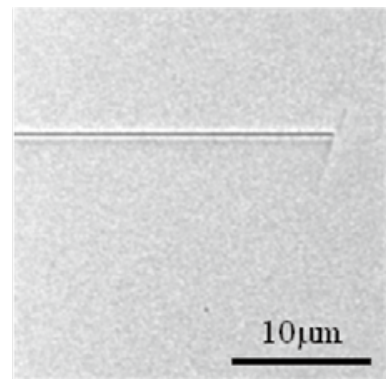

(e)

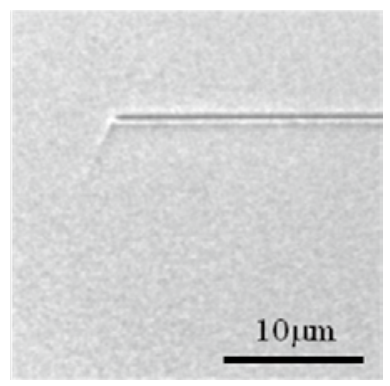

(f)

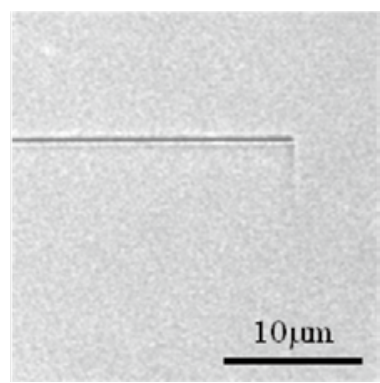

(g)

Fig. 7. Assessment results by MEM at measurement points: (a) point a, (b) point b, (c) point c, (d) point $\mathrm{d}$, (e) point e, (f) point h, and (g) point i.

Figure 9 shows the MEM assessment results for the defect along $\mathrm{f}-\mathrm{g}$, which was detected by PL spectroscopy but not by X-ray topography. The MEM assessment results are the same as the results for the other measurement points. These results suggest that the defect along $\mathrm{f}-\mathrm{g}$ is a stacking fault. 




(a)

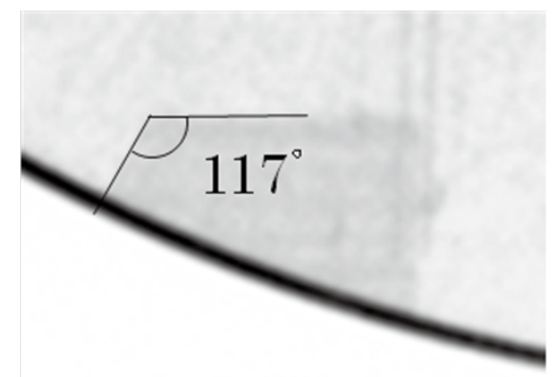

(b)

Fig. 8. Incursion angle to substrate for stacking fault at measurement point h: (a) incursion angle according to MEM assessment and (b) incursion angle according to X-ray topography assessment.

Table 1

Measurement results of incursion angle to substrate for stacking faults.

\begin{tabular}{ccc}
\hline \multirow{2}{*}{ Position } & \multicolumn{2}{c}{ Incursion angle to substrate interior $\left[{ }^{\circ}\right]$} \\
\cline { 2 - 3 } & MEM & X-ray topography \\
\hline a & 146 & 148 \\
$\mathrm{~b}$ & 41 & 43 \\
$\mathrm{c}$ & 59 & 60 \\
$\mathrm{~d}$ & 115 & 116 \\
$\mathrm{e}$ & 75 & 73 \\
$\mathrm{~h}$ & 116 & 117 \\
$\mathrm{i}$ & 88 & 86 \\
\hline
\end{tabular}

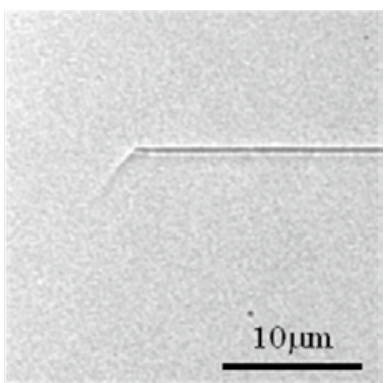

(a)

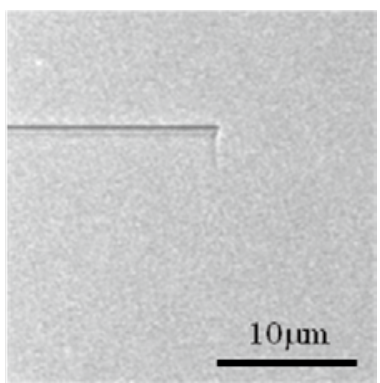

(b)

Fig. 9. Assessment results by MEM at measurement points: (a) point $f$ and (b) point $g$. 
Figure 10 shows the results of analysis of point $\mathrm{f}$ with and without UV irradiation. Compared with the non-UV irradiation case shown in Fig. 10(a), the UV irradiation case shown in Fig. 10(b) provides a clearer assessment of the stacking fault. This clarity is thought to be due to carriers generated by UV irradiation being trapped by the stacking fault, which alters the detected electric potential.

Figure 11 shows the results of assessment by MEM for a defect that is not a stacking fault; a defect intersecting a stacking fault was detected. This defect was not a uniform line, as was observed for stacking faults. It is thought to be a scratch created during mirror polishing of the wafer surface. However, another evaluation is necessary to obtain a detailed profile of these defects. In this way, MEM can be used to assess various defects arising from potential differences and level differences on the surface.

\subsection{AFM assessment}

Figure 12 shows AFM analysis results for point h. AFM assessment revealed the presence of level differences on the top surface for stacking faults. AFM analysis also



(a)

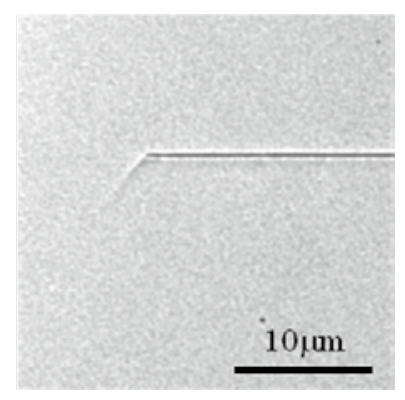

(b)

Fig. 10. MEM assessment results: (a) with and (b) without $\mathrm{UV}$ radiation at measurement point $\mathrm{f}$.

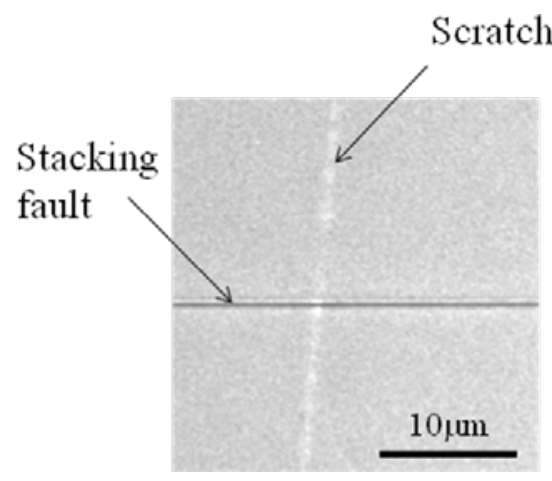

(a)



(b)

Fig. 11. MEM assessment results: (a) between $\mathrm{h}$ and $\mathrm{i}$ and (b) between $\mathrm{f}$ and $\mathrm{g}$. 




(a)



(b)

Fig. 12. AFM assessment results at measurement point h: (a) two-dimensional scan and (b) incursion angle.

enabled the detection of a stacking fault with an incursion to the interior of a wafer. As shown in Fig. 12(b), the incursion angle was $116^{\circ}$, which is the same value as the assessment results obtained by X-ray topography and MEM.

A quantitative assessment of level differences is provided by the results in Fig. 13 for AFM analysis. Figure 13(a) shows the analysis site. Figure 13(b) shows the results of a simple, one-dimensional scan. The level differences are extremely small, being on the order of atomic steps. Figure 13(c) shows the results obtained by integrating measured values across a width of about $2 \mu \mathrm{m}$ and averaging. They reveal level differences on the outermost front surface for the stacking faults to be about $0.1 \mathrm{~nm}$. The wafer used for assessment was mirror polished. The chemical etch rate is slightly higher in the section of stacking faults. There is thus a high probability that this higher etch rate generates level differences on the wafer surface.

\section{Summary}

X-ray topography, PL spectroscopy, MEM, and AFM were employed to assess stacking faults in $\mathrm{SiC}$ crystals. Transmission X-ray topography was found to be capable of assessing stacking faults of crystal interiors. PL spectroscopy and MEM were found to be capable of assessing stacking faults exposed on crystal surfaces. One stacking fault that was detected by PL spectroscopy and MEM was undetected by X-ray topography. This defect requires further detailed assessment. The stacking faults assessed by the various methods were the same. In addition, assessment by AFM revealed the presence of microscopic level differences of about $0.1 \mathrm{~nm}$ in regions where stacking faults were exposed on the surface. These level differences are thought to arise from differences in the etch rate during mirror polishing. 




(b)

(a)

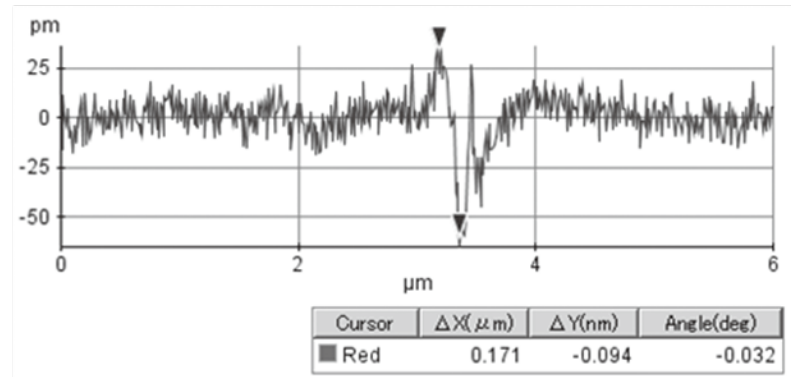

(c)

Fig. 13. Level difference measurement by AFM: (a) analysis site, (b) simple, one-dimensional scan, and (c) analytical results of integration.

\section{Acknowledgements}

The author gratefully acknowledges assistance with the X-ray topography analysis from Mr. Toshiro Futagi of Nippon Steel Technoresearch Corp., with PL spectroscopy from Prof. Atsushi Ogura of Meiji University and Mr. Ichiro Chiba of Photon Design Corp., with MEM analysis from Hitachi High-Technologies Corporation and Hitachi, Ltd., Central Research Laboratory, and with AFM analysis from Park Systems Corp.

\section{References}

1 Y. M. Tairov: The 9th Eur. Conf. on Silicon Carbide and Related Materials (ECSCRM 2012, Saint-Petersburg, Russia) Book of Abstracts Mo1-1.

2 L. Ottaviani, S. Biondo, W. Vervisch, M. Lazar, A. Lyoussi, F. Milesi, O. Palais and F. Torregrosa: The 9th Eur. Conf. on Silicon Carbide and Related Materials (ECSCRM 2012, Saint-Petersburg, Russia) Book of Abstracts Th11-1.

3 H. Yamamoto and T. Hashizume: Phys. Status Solidi C C8 (2011) 82.

4 T. A. Kuhr, J. Q. Liu, H. J. Chung, M. Skowronski and F. Szmulowicz: J. Appl. Phys. 92 (2002) 5863. 
5 M. Hasegawa and T. Shimakura: J. Appl. Phys. 107 (2010) 084107.

6 S. Izumi, H. Tsuchida, I. Kamata and T. Tawara: Appl. Phys. Lett. 86 (2005) 202108.

\section{About the Author}



Hidekazu Yamamoto received the B.E., M.E., and Ph.D. degrees in electrical engineering from Hokkaido University, in 1979, 1981, and 1984, respectively. In 1984, he joined the Mitsubishi Electric Corporation, where he engaged in the process and material technologies for LSIs and power devices. In 2010, he joined Chiba Institute of Technology, he has been investigated the crystal for power devices. He is a member of the Japan Society of Applied Physics, the Institute of Electrical Engineering of Japan, and the Institute of Electronics, Information and Communication Engineers. 